\title{
Corneal subbasal nerve plexus changes in patients with episodic migraine: an in vivo confocal microscopy study
}

This article was published in the following Dove Press journal: Journal of Pain Research

\author{
Feifei Shen ${ }^{1} *$ \\ Xin Dong ${ }^{1, *}$ \\ Xin Zhou ${ }^{2}$ \\ Lanyun Yan' \\ Qi Wan'
}

'Department of Neurology, The First Affiliated Hospital of Nanjing Medical University, Nanjing, Jiangsu, 210029 ,

People's Republic of China; ${ }^{2}$ Department of Ophthalmology, The Affiliated Hospital of Nanjing University of Traditional Chinese Medicine, Nanjing, Jiangsu 210029, People's Republic of China

*These authors contributed equally to this work
Correspondence: Qi Wan; Lanyun Yan Department of Neurology, The First Affiliated Hospital of Nanjing Medical University, Nanjing, Jiangsu 210029, People's Republic of China

Tel +86I 37703 I 6989.

$+8615850515714$

Fax +860 258 371 8836

Email qi_wan@I26.com;

yan_lanyun@I26.com
Background and purpose: It has been generally thought that activation and sensitization of the trigeminovascular system may contribute to the pathogenesis of migraine. Nevertheless, there is little evidence on abnormalities in peripheral trigeminal afferent nerves from humans in vivo. Alterations of corneal nerves from the ophthalmic branch of the trigeminal nerve may support the notion that trigeminal afferent nerves are involved in migraine pathophysiology. The aim of the present study was to investigate the structural changes in corneal subbasal nerve plexus in patients with episodic migraine (EM) with in vivo confocal microscope (IVCM).

Methods: In this cross-sectional observational study, 10 EM patients and 10 age- and sexmatched healthy controls were included. Analysis of IVCM images with Image J software was performed to quantify the changes in the corneal subbasal nerve plexus.

Results: EM patients showed an increase in nerve fiber length $(25.0 \pm 2.65 \mathrm{vs} 22.3 \pm 2.41 \mathrm{~mm} /$ $\left.\mathrm{mm}^{2}, p=0.047\right)$ and nerve fiber density $\left(36.3 \pm 7.29\right.$ vs $30.5 \pm 6.19$ fibers $\left./ \mathrm{mm}^{2}, p=0.104\right)$ as compared with normal controls, but this difference was not statistically significant. Nerve branching and tortuosity were significantly increased in the EM subjects compared to the normal subjects $\left(91.3 \pm 13.8\right.$ vs $75.0 \pm 14.2$ branches $/ \mathrm{mm}^{2}, p=0.030$ and $2.30 \pm 0.46$ versus 1.63 $\pm 0.52, p=0.011$, respectively). In addition, nerve sprouts and increased number of Langerhans cells were observed in the EM patients.

Conclusion: The morphologic changes of corneal subbasal nerve plexus and Langerhans cell aggregation suggest the presence of nerve regeneration and inflammation in EM. Furthermore, the alterations of corneal nerves from the ophthalmic branch of the trigeminal nerve offer support for the hypothesis that the peripheral trigeminal system may be involved in the pathogenesis of migraine.

Keywords: migraine, corneal nerves, subbasal nerve plexus, in vivo confocal microscopy

\section{Introduction}

Migraine is a common disabling neurovascular disorder characterized by severe unilateral throbbing headache accompanied by nausea, vomiting, photophobia and phonophobia. ${ }^{1}$ Based on attack frequency, migraine sub-classified into episodic migraine (EM) and chronic migraine (CM). Patients with $<15$ days of headache per month are defined as EM, and those with headache occurring on more than 15 days per month, which has features of migraine headache on at least 8 days, are diagnosed as CM. Although the pathophysiology of migraine has not been fully understood, it is generally thought that activation and sensitization of the 
trigeminovascular system is responsible for migraine attack. $^{2,3}$ So far, most of the evidence has come from animal studies, and relatively few in vivo human studies using functional imaging tend to support the hypotheses. Electrophysiological recordings showed that topical application of inflammatory soup can activate and sensitize the trigeminal ganglion, the trigeminal spinal nucleus and thalamus in the rats. ${ }^{4-6}$ Evidence from human functional imaging studies also showed activation of the peripheral and central trigeminal system in migraine. ${ }^{7,8}$ Nevertheless, there is little evidence on abnormalities in peripheral trigeminal afferent nerves from humans in vivo.

Most corneal nociceptive afferents are derived from the ophthalmic branch of the trigeminal nerve and project to the trigeminal nuclear complex. ${ }^{9}$ Alterations of corneal nerves may support the role of trigeminal afferent nerves in the physiopathology of migraine. Photophobia is a common symptom in migraine and has been identified as one of the diagnostic criteria of migraine by the International Classification of Headache Disorders. The prevalence of photophobia is approximately $80 \%$ of migraine sufferers. ${ }^{10}$ The symptom of dry eye is also extremely prevalent in CM. ${ }^{11}$ These ocular symptoms associated with migraine indicate the possibility of changes in the corneal axons. Consistently, previous studies have demonstrated that there are significant structural changes in the subbasal corneal nerve plexus in patients with CM. ${ }^{11,12}$ Nevertheless, the structural alterations of corneal afferents in EM remain unknown. Early identification of the abnormalities in corneal afferent is pivotal for the understanding of migraine pathogenesis and for the early intervention of ocular symptoms.

In vivo confocal microscopy (IVCM) is a noninvasive technique for the examination of the cornea. It is widely used in the study of corneal subbasal nerve plexus in various ocular and systemic diseases. ${ }^{13,14}$ We hypothesized that the alterations of corneal afferent nerves could participate in the pathogenesis of EM. In the present study, we aimed to investigate the structural changes of corneal subbasal nerve plexus in patients with EM with IVCM.

\section{Methods}

\section{Participants}

This cross-sectional observational study was approved by the Ethics Committee of the First Affiliated Hospital of Nanjing Medical University and was carried out in accordance with the principles of the Declaration of Helsinki.
Written informed consent was obtained from all patients. Ten EM patients were recruited from the outpatients in Neurology Clinic of the First Affiliated Hospital of Nanjing Medical University between May 1, 2017 and December 31, 2017. The diagnosis of migraine was made according to the International Classification of Headache Disorders criteria. Inclusion criteria were the presence of EM with $<15$ headache days per month. Patients with peripheral neuropathy and ocular diseases were excluded. We also excluded the possible small fiber neuropathic patients presenting with length-dependent neuropathic pain and/or clinical signs of small-fiber damage according to international diagnostic criteria. ${ }^{15}$ We did not exclude migraine subjects with dry eye disease that may be associated with migraine. The control group included 10 age- and sex-matched healthy subjects.

\section{Confocal microscopy}

Each eye was examined with a laser scanning confocal microscope (Heidelberg Retina Tomograph $\square$ Rostock Corneal Module; Heidelberg Engineering $\mathrm{GmbH}$, Heidelberg, Germany). IVCM was performed under topical anesthesia with one drop of $0.4 \%$ oxybuprocaine hydrochloride (Santen Ltd, Japan). A TomoCap (Heidelberg Engineering $\mathrm{GmbH}$, Heidelberg, Germany) was used as the contact cap, and a drop of $0.2 \%$ sterile ophthalmic gel (Bausch \& Lomb Ltd; Germany) was used as coupling medium between the contact cap and objective lens of the microscope. The subjects were asked to fixate on a blinking target as the examination was performed. Central regions of the cornea were scanned through all the corneal layers.

\section{Image analysis}

The field size examined by confocal microscope was $400 \times 400 \mu \mathrm{m}$. One eye of each subject was selected at random for image analysis. Three high-quality images were collected from the center of the cornea for corneal subbasal nerve plexus assessments. Quantitative analysis of the corneal nerve fibers was performed with Image $\mathrm{J}$ analysis software, version 1.8.0 (National Institutes of Health, Bethesda, MD, USA) by a trained examiner who was blind to the grouping of the participants throughout the study. Parameters of corneal subbasal nerve plexus quantified in the image frame included: nerve fiber length (NFL), defined as the total length of all nerve fibers per square millimeter; nerve fiber density (NFD), defined as the total number of major nerve fibers per square millimeter; nerve branch density (NBD), defined as the 
number of branches per square millimeter; tortuosity, graded from 0 to 4 following the Oliveira-Soto scale. ${ }^{16}$

\section{Statistical analysis}

All the data were presented as the mean $\pm \mathrm{SD}$. The demographic characteristics between migraine patients and normal controls were compared using Student'st-test for continuous variables and Fisher's exact test for categorical variables. The intergroup differences in outcome parameters were analyzed with the $t$-test. Differences with $p<0.05$ were considered statistically significant. The statistical analysis was performed using SPASS software, version 21.0 (SPASS Inc, Chicago, IL, USA).

\section{Results}

All the migraineurs and controls completed the study and were included in the analyses. The demographic and clinical characteristics are shown in Table 1 . The study included 10 EM patients with or without aura and 10 healthy subjects as controls. There was no significant difference in mean age and sex between the migraine patients and control groups. The mean age was $38.9 \pm 6.31$ years in migraine subjects, and $37.3 \pm 5.54$ years in control participants. The migraine patients reported a mean headache frequency of $4.11 \pm 2.57$ days/month and a mean pain intensity of $8.00 \pm 1.58$ evaluated by a $0-10$ scale.

Representative images of the corneal subbasal nerve plexus in the normal subjects and migraine patients are presented in Figure 1. The corneal subbasal nerve plexus in the control individuals revealed parallel appearance with slight tortuosity and branches (Figure 1A). In

Table I Demographic and clinical characteristics of EM patients and control subjects

\begin{tabular}{|c|c|c|c|}
\hline Parameters & $\begin{array}{l}\text { Migraine } \\
(n=10)\end{array}$ & $\begin{array}{l}\text { Control } \\
(n=10)\end{array}$ & $\boldsymbol{p}$ \\
\hline Age (years) & $38.9 \pm 6.31$ & $37.3 \pm 5.54$ & 0.586 \\
\hline Sex (female/male) & $9 / 1$ & $8 / 2$ & 1.00 \\
\hline $\begin{array}{l}\text { Migraine history } \\
\text { (years) }\end{array}$ & $14.4 \pm 5.90$ & $\mathrm{~N} / \mathrm{A}$ & $\mathrm{N} / \mathrm{A}$ \\
\hline $\begin{array}{l}\text { Attack frequency per } \\
\text { month }\end{array}$ & $4.11 \pm 2.57$ & $N / A$ & N/A \\
\hline Pain intensity & $8.00 \pm 1.58$ & N/A & N/A \\
\hline Attack duration (hrs) & $36.6 \pm 21.5$ & N/A & $\mathrm{N} / \mathrm{A}$ \\
\hline Aura & $10 \%$ & N/A & $\mathrm{N} / \mathrm{A}$ \\
\hline Photophobia & $80 \%$ & $\mathrm{~N} / \mathrm{A}$ & $\mathrm{N} / \mathrm{A}$ \\
\hline
\end{tabular}

Note: Data shown as mean $\pm S D, n / n$, and $\%$.

Abbreviations: EM, episodic migraine; N/A, not available. comparison, the corneal subbasal nerves in many of the EM patients revealed increased nerve tortuosity and branches (Figure 1B). In addition, nerve sprouts were observed in $20 \%$ of the migraine patients (Figure 1C) and increased number of Langerhans cells were present in most of the migraine patients (Figure 1D).

The results of corneal subbasal nerve measurements are summarized in Table 2. EM patients showed an increase in NFL $\left(25.0 \pm 2.65\right.$ vs $22.3 \pm 2.41 \mathrm{~mm} / \mathrm{mm}^{2}$, $p=0.047)$ and NFD (36.3 \pm 7.29 vs $30.5 \pm 6.19$ fibers $/ \mathrm{mm}^{2}$, $p=0.104)$ as compared with the control group, but this difference was not statistically significant. There was a significant difference in NBD between the migraine group and the control group $(91.3 \pm 13.8$ vs $75.0 \pm 14.2$ branches $/ \mathrm{mm}^{2}, p=0.030$ ). Nerve tortuosity was also significantly higher in the migraine subjects than in normal subjects $(2.30 \pm 0.46$ versus $1.63 \pm 0.52, p=0.011)$.

\section{Discussion}

In the present study, ICVM was used to detect the morphologic alterations of corneal subbasal nerve plexus in patients with EM. The corneal subbasal nerves in EM patients showed increased nerve length, density, branches and tortuosity compared with normal controls. In addition, nerve sprouts and increased number of Langerhans cells were observed in EM patients.

IVCM has been widely used to image and quantify the corneal subbasal nerve plexus in various eye conditions, such as dry eye and photophobia associated with migraine. ${ }^{14}$ Our study is the first to study the morphologic alterations of corneal subbasal nerve plexus in EM. A previous study conducted by Kinard et al showed that the nerve density and length was significantly decreased in patients with $\mathrm{CM}$ compared with control subjects. ${ }^{11}$ In a recent study, Shetty et al also found a significant decrease in the corneal subbasal nerve length and NBD. ${ }^{12}$ These morphologic changes of corneal subbasal nerve fibers in CM shown in these previous studies may relate to impairment or degeneration of corneal nerves, which were inconsistent with our present results observed in EM. The increased nerve density, length and branches in EM suggest the occurrence of corneal nerve regeneration. Similar to our findings, regeneration of corneal nerves has also been observed in dry eye condition due to Sjogren's disease syndrome. ${ }^{17}$ It can be inferred that regeneration of corneal nerve may occur as a feedback from the slight nerve degeneration or injury when the migraine attack is infrequent, whereas maladaptive changes of severe neural 

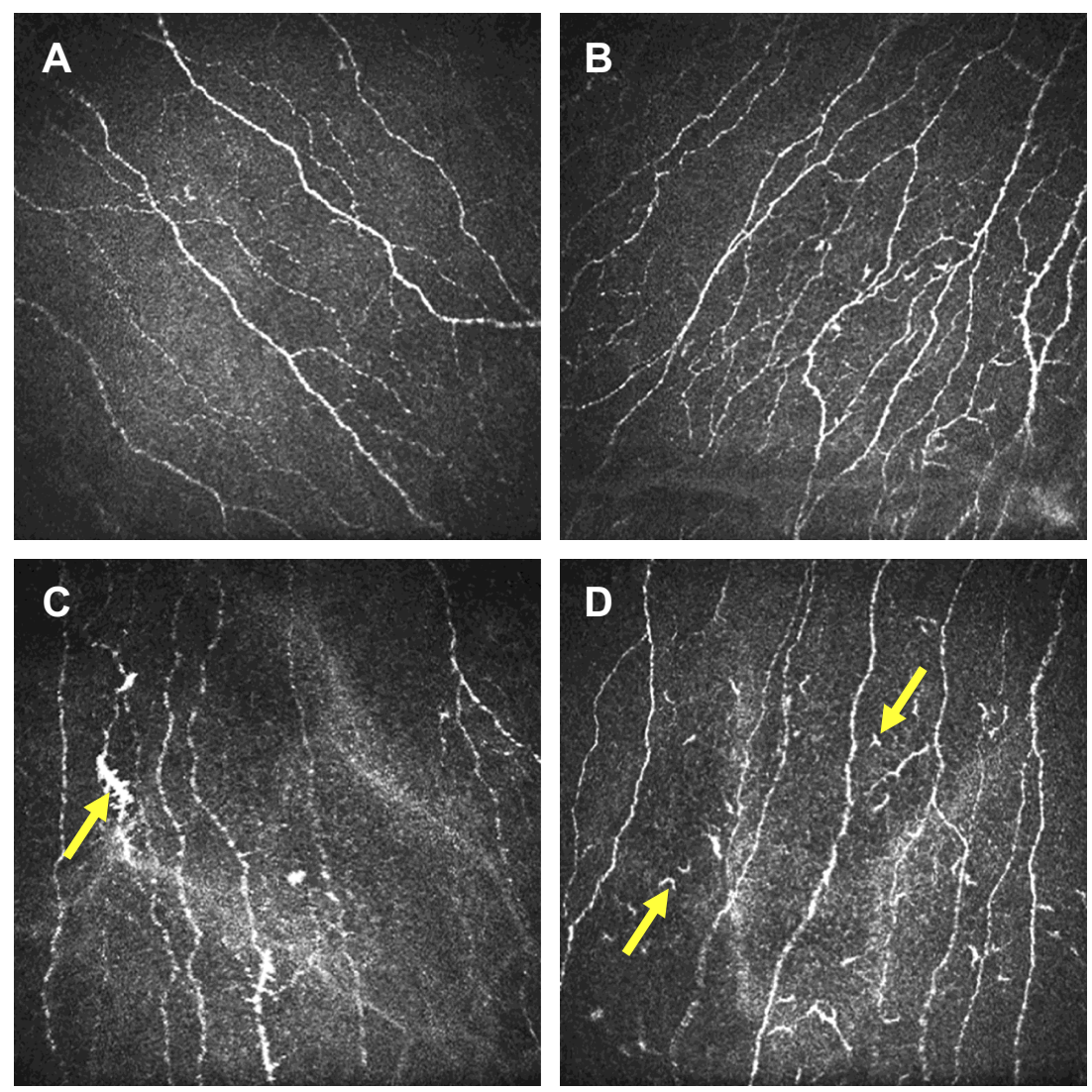

Figure I Representative images of the corneal subbasal nerves in normal subjects and migraine patients. (A) Image of the corneal subbasal nerves in a normal subject. (B) Image of the corneal subbasal nerves in an EM subject with increased nerve tortuosity and branches. (C) Image of the corneal subbasal nerves in an EM subject with nerve sprouts. (D) The arrows highlight the increased number of Langerhans cells in the corneal subbasal nerves in an EM subject. Images were $400 \times 400 \mu \mathrm{m}$.

Abbreviation: EM, episodic migraine.

Table 2 Subbasal corneal nerve measurements in EM patients and control subjects

\begin{tabular}{|c|c|c|c|}
\hline Parameters & Migraine $(n=10)($ mean $\pm S D)$ & Control $(n=10)($ mean $\pm S D)$ & $\mathbf{P}$ \\
\hline NFD (number/mm²) & $36.3 \pm 7.29$ & $30.5 \pm 6.19$ & 0.104 \\
\hline $\mathrm{NFL}\left(\mathrm{mm} / \mathrm{mm}^{2}\right)$ & $25.0 \pm 2.65$ & $22.3 \pm 2.41$ & 0.047 \\
\hline NBD (number/mm²) & $91.3 \pm 13.8$ & $75.0 \pm 14.2$ & $0.030 *$ \\
\hline Tortuosity & $2.30 \pm 0.46$ & $1.63 \pm 0.52$ & $0.011 *$ \\
\hline
\end{tabular}

Note: ${ }^{*} p<0.05$

Abbreviations: EM, episodic migraine; NFD, nerve fiber density; NFL, nerve fiber length; NBD, nerve branch density.

degeneration occur later with the chronicity of migraine. Therefore, the occurrence of neural regeneration or degeneration suggests adaptive or dysregulated mechanisms in episodic or CM.

The present results suggest the regeneration of corneal nerves in EM patients. ${ }^{14}$ Presumably, it may be the subsequent feedback from the nerve injury or degeneration. The morphological characteristics of corneal nerve regeneration remain to be clearly elucidated. ${ }^{17-20}$ In the present study, increased nerve density, length, branching and tortuosity were observed in the EM patients, indicating an attempt of corneal subbasal nerves to regenerate. Additionally, the presence of nerve sprouts in corneal subbasal nerves in migraine patients also serves as a morphologic marker of nerve regeneration. ${ }^{21,22}$ The mechanism for the regeneration of corneal nerves in migraine patients is still unclear. Increased peripheral serum levels of nerve growth factor (NGF) detected in patients with migraine may be responsible for the corneal regeneration. ${ }^{23,24}$ Peptidergic nerves containing peptides 
such as calcitonin gene-related peptide (CGRP) and substance $\mathrm{P}$ have been described in the cornea. ${ }^{25,26}$ It is generally recognized that activation of the trigeminal nociceptive afferents can trigger the release of these vasoactive peptides in trigeminal nerve endings, leading to neurogenic inflammation. ${ }^{27}$ It has been shown that salivary levels of CGRP are significantly increased between headache attacks in migraine patients compared with control subjects. Neurogenic inflammation can lead to the release of proinflammatory cytokines, such as IL-1 and IL- $6 .{ }^{28}$ The release of peptides and proinflammatory cytokines can subsequently induce the synthesis of NGF and other factors with a neurotrophic effect. ${ }^{29,30}$ Overexpression of NGF stimulates the nerve growth and regeneration. ${ }^{31,32}$

Langerhans cells are a subset of antigen-presenting cells that locate in the epidermal layer of the skin. ${ }^{33}$ Langerhans cells also reside in the normal human cornea, and inflammation can induce increased corneal Langerhans cells in the cornea. ${ }^{34,35}$ The present study observed Langerhans cell aggregation in corneal subbasal nerves in EM patients, which indicated a peripheral inflammation-immune process of the trigeminal system. Previous studies have shown that dendritic cells play a role in the modulation of nociception and pain through their effect on $\mathrm{T}$ cells. ${ }^{36}$ The dendritic cell-mediated inflammatory irritation of the nerve may be involved in the pathophysiology of migraine, which warrants further investigation.

Ocular discomfort caused by light exposure in migraine is proposed to involve activation of ocular nociceptors that project to the spinal trigeminal nucleus through the trigeminal nerve..$^{37,38}$ The morphologic changes and inflammation in corneal nerves in migraineurs in our study may initiate the pathophysiology of ocular discomfort caused by light exposure. In addition, the abnormalities in corneal nerves involved in the lacrimal function unit may disrupt the normal neural feedback ${ }^{17,39}$ and result in dry eye condition in migraine. Early identification of the abnormalities in corneal nerves is critical for the understanding of the pathogenesis of ocular symptoms associated with migraine and for the early intervention of ocular symptoms. Additionally, the changes in the corneal nerves support the role of the trigeminal system in the pathogenesis of migraine. Nevertheless, it is impossible to draw a conclusion whether these morphologic changes of corneal subbasal nerves cause migraine by activation of the first branch of the trigeminal nerve or whether migraine results in such changes of corneal subbasal nerves based on the present results. A limitation of our present study is the small sample size. Further studies with larger sample size are needed to obtain less risk for Type II errors and more chances to detect a significant difference when it exists. Furthermore, corneal nerves contain both sensory fibers and autonomic sympathetic nerve fibers. Thus, it is difficult to determine if it is the nociceptive fibers that undergo structural changes. Further anatomic and immunohistological studies in animal models are needed to elucidate this issue.

\section{Conclusion}

In conclusion, IVCM provides a rapid and noninvasive technique to quantify the corneal subbasal nerve plexus in patients with EM. The morphologic changes of corneal subbasal nerves and Langerhans cell aggregation indicate the presence of nerve regeneration and inflammation in EM. Furthermore, the alterations of corneal nerve from the ophthalmic branch of the trigeminal nerve offer support for the hypothesis that the peripheral trigeminal system may play a role in the pathogenesis of migraine. Further investigations are required to define the relationship between corneal subbasal nerve changes and migraine pathophysiology.

\section{Acknowledgment}

This work was supported by the National Natural Science Foundation of China (grant number 81600970).

\section{Author contributions}

All authors contributed toward data analysis, drafting and critically revising the paper, gave final approval of the version to be published, and agree to be accountable for all aspects of the work.

\section{Disclosure}

The authors report no conflicts of interest in this work.

\section{References}

1. Arnold M. Headache classification committee of the international headache society (IHS) the international classification of headache disorders, 3rd edition. Cephalalgia. 2018;38(1):1-211. doi:10.1177/ 0333102417738202

2. Noseda R, Burstein R. Migraine pathophysiology: anatomy of the trigeminovascular pathway and associated neurological symptoms, CSD, sensitization and modulation of pain. Pain. 2013;154(Suppl):1. doi:10.1016/j.pain.2013.07.021

3. Charles A. The pathophysiology of migraine: implications for clinical management. Lancet Neurol. 2018;17(2):174-182. doi:10.1016/ S1474-4422(17)30435-0 
4. Strassman AM, Raymond SA, Burstein R. Sensitization of meningeal sensory neurons and the origin of headaches. Nature. 1996;384 (6609):560-564. doi:10.1038/384560a0

5. Burstein R, Yamamura H, Malick A, Strassman AM. Chemical stimulation of the intracranial dura induces enhanced responses to facial stimulation in brain stem trigeminal neurons. J Neurophysiol. 1998;79(2):964-982. doi:10.1152/jn.1998.79.2.964

6. Burstein R, Jakubowski M, Garcia-Nicas E, et al. Thalamic sensitization transforms localized pain into widespread allodynia. Ann Neurol. 2010;68(1):81-91. doi:10.1002/ana.21994

7. Borsook D, Burstein R, Becerra L. Functional imaging of the human trigeminal system: opportunities for new insights into pain processing in health and disease. J Neurobiol. 2004;61(1):107-125. doi:10.1002/ neu. 20085

8. Schwedt TJ, Chiang CC, Chong CD, Dodick DW. Functional MRI of migraine. Lancet Neurol. 2015;14(1):81-91. doi:10.1016/S14744422(14)70193-0

9. Muller LJ, Marfurt CF, Kruse F, Tervo TM. Corneal nerves: structure, contents and function. Exp Eye Res. 2003;76(5):521-542.

10. Choi JY, Oh K, Kim BJ, Chung CS, Koh SB, Park KW. Usefulness of a photophobia questionnaire in patients with migraine. Cephalalgia. 2009;29(9):953-959. doi:10.1111/j.1468-2982.2008.01822.x

11. Kinard KI, Smith AG, Singleton JR, et al. Chronic migraine is associated with reduced corneal nerve fiber density and symptoms of dry eye. Headache. 2015;55(4):543-549. doi:10.1111/head.12547

12. Shetty R, Deshmukh R, Shroff R, Dedhiya C, Jayadev C. Subbasal nerve plexus changes in chronic migraine. Cornea. 2018;37 (1):72-75. doi:10.1097/ICO.0000000000001403

13. Cruzat A, Pavan-Langston D, Hamrah P. In vivo confocal microscopy of corneal nerves: analysis and clinical correlation. Semin Ophthalmol. 2010;25(5-6):171-177. doi:10.3109/08820538.2010.518133

14. Cruzat A, Qazi Y, Hamrah P. In vivo confocal microscopy of corneal nerves in health and disease. Ocul Surf. 2017;15(1):15-47. doi:10.1016/j.jtos.2016.09.004

15. Sopacua M, Hoeijmakers JGJ, Merkies ISJ, Lauria G, Waxman SG, Faber CG. Small-fiber neuropathy: expanding the clinical pain universe. J Peripher Nerv Syst. 2018;24(1):19-33.

16. Oliveira-Soto L, Efron N. Morphology of corneal nerves using confocal microscopy. Cornea. 2001;20(4):374-384.

17. Zhang M, Chen J, Luo L, Xiao Q, Sun M, Liu Z. Altered corneal nerves in aqueous tear deficiency viewed by in vivo confocal microscopy. Cornea. 2005;24(7):818-824.

18. Kawabuchi M, Chongjian Z, Islam AT, Hirata K, Nada O. The effect of aging on the morphological nerve changes during muscle reinnervation after nerve crush. Restor Neurol Neurosci. 1998;13(3-4):117-127.

19. Muller RT, Abedi F, Cruzat A, et al. Degeneration and regeneration of subbasal corneal nerves after infectious keratitis: a longitudinal in vivo confocal microscopy study. Ophthalmology. 2015;122 (11):2200-2209. doi:10.1016/j.ophtha.2015.06.047

20. Moein HR, Kheirkhah A, Muller RT, Cruzat AC, Pavan-Langston D, Hamrah P. Corneal nerve regeneration after herpes simplex keratitis: A longitudinal in vivo confocal microscopy study. Ocul Surf. 2018;16 (2):218-225. doi:10.1016/j.jtos.2017.12.001

21. Tuominen IS, Konttinen YT, Vesaluoma MH, Moilanen JA, Helinto M, Tervo TM. Corneal innervation and morphology in primary Sjogren's syndrome. Invest Ophthalmol Vis Sci. 2003;44 (6):2545-2549.

22. Benitez Del Castillo JM, Wasfy MA, Fernandez C, Garcia-Sanchez J. An in vivo confocal masked study on corneal epithelium and subbasal nerves in patients with dry eye. Invest Ophthalmol Vis Sci. 2004;45(9):3030-3035. doi:10.1167/iovs.04-0251
23. Jang MU, Park JW, Kho HS, Chung SC, Chung JW. Plasma and saliva levels of nerve growth factor and neuropeptides in chronic migraine patients. Oral Dis. 2011;17(2):187-193. doi:10.1111/j.16010825.2010.01717.x

24. Martins LB, Teixeira AL, Domingues RB. Neurotrophins and migraine. Vitam Horm. 2017;104:459-473. doi:10.1016/bs. vh.2016.10.003

25. Jones MA, Marfurt CF. Peptidergic innervation of the rat cornea. Exp Eye Res. 1998;66(4):421-435. doi:10.1006/exer.1997.0446

26. He J, Bazan HE. Neuroanatomy and neurochemistry of mouse cornea. Invest Ophthalmol Vis Sci. 2016;57(2):664-674. doi:10.1167/iovs.15-18019

27. Levy D, Labastida-Ramirez A, MaassenVanDenBrink A. Current understanding of meningeal and cerebral vascular function underlying migraine headache. Cephalalgia. Epub Jan 2018.

28. Zhang X, Burstein R, Levy D. Local action of the proinflammatory cytokines IL-1beta and IL-6 on intracranial meningeal nociceptors. Cephalalgia. 2012;32(1):66-72. doi:10.1177/ 0333102411430848

29. Dallos A, Kiss M, Polyanka H, Dobozy A, Kemeny L, Husz S. Effects of the neuropeptides substance $\mathrm{P}$, calcitonin gene-related peptide, vasoactive intestinal polypeptide and galanin on the production of nerve growth factor and inflammatory cytokines in cultured human keratinocytes. Neuropeptides. 2006;40(4):251-263. doi:10.1016/j.npep.2006.06.002

30. Buldyrev I, Tanner NM, Hsieh HY, Dodd EG, Nguyen LT, Balkowiec A. Calcitonin gene-related peptide enhances release of native brain-derived neurotrophic factor from trigeminal ganglion neurons. J Neurochem. 2006;99(5):1338-1350. doi:10.1111/j.14714159.2006.04161.x

31. Di G, Qi X, Zhao X, Zhang S, Danielson P, Zhou Q. Corneal epithelium-derived neurotrophic factors promote nerve regeneration. Invest Ophthalmol Vis Sci. 2017;58(11):4695-4702. doi:10.1167/ iovs.16-21372

32. Sacchetti M, Lambiase A. Neurotrophic factors and corneal nerve regeneration. Neural Regen Res. 2017;12(8):1220-1224. doi:10.4103/1673-5374.213534

33. Merad M, Ginhoux F, Collin M. Origin, homeostasis and function of Langerhans cells and other langerin-expressing dendritic cells. Nat Rev Immunol. 2008;8(12):935-947. doi:10.1038/nri2455

34. Mastropasqua L, Nubile M, Lanzini M, et al. Epithelial dendritic cell distribution in normal and inflamed human cornea: in vivo confocal microscopy study. Am J Ophthalmol. 2006;142(5):736-744. doi:10.1016/j.ajo.2006.06.057

35. Hattori T, Takahashi H, Dana R. Novel insights into the immunoregulatory function and localization of dendritic cells. Cornea. 2016;35(Suppl 1):S49-S54. doi:10.1097/ICO.0000000000 001005

36. Luo J, Feng J, Liu S, Walters ET, Hu H. Molecular and cellular mechanisms that initiate pain and itch. Cell Mol Life Sci. 2015;72 (17):3201-3223. doi:10.1007/s00018-015-1904-4

37. Okamoto K, Tashiro A, Chang Z, Bereiter DA. Bright light activates a trigeminal nociceptive pathway. Pain. 2010;149(2):235-242. doi:10.1016/j.pain.2010.02.004

38. Noseda R, Burstein R. Advances in understanding the mechanisms of migraine-type photophobia. Curr Opin Neurol. 2011;24(3):197-202. doi:10.1097/WCO.0b013e3283466c8e

39. Stern ME, Beuerman RW, Fox RI, Gao J, Mircheff AK, Pflugfelder SC. The pathology of dry eye: the interaction between the ocular surface and lacrimal glands. Cornea. 1998;17(6):584-589. 


\section{Publish your work in this journal}

The Journal of Pain Research is an international, peer reviewed, open access, online journal that welcomes laboratory and clinical findings in the fields of pain research and the prevention and management of pain Original research, reviews, symposium reports, hypothesis formation and commentaries are all considered for publication. The manuscript management system is completely online and includes a very quick and fair peer-review system, which is all easy to use. Visit http:// www.dovepress.com/testimonials.php to read real quotes from published authors.

Submit your manuscript here: https://www.dovepress.com/journal-of-pain-research-journal 